\section{TATRA \\ MOUNTaiNS \\ Mathematical Publications}

DOI: $10.1515 / \mathrm{tmmp}-2016-0029$

Tatra Mt. Math. Publ. 67 (2016), 47-53

\title{
SUMS OF GOLDEN ANA SETS
}

\author{
ROBERT W. VALLIN
}

\begin{abstract}
It is well-known that despite being a small set in the unit interval, the sum of a Cantor set and itself is the interval $[0,2]$. In this paper we look for an analogous result on a different small set, the Golden Ana set.
\end{abstract}

\section{Introduction}

The Cantor set $C$ is a favorite example for different types of small set (e.g., measure zero, nowhere dense). One of its more surprising properties is that the sum of this set and itself

$$
C+C=\{x+y \mid x, y \in C\}
$$

returns the interval $[0,2]$. There are several proofs of it, one favorite is the proof depicted in the Fig. 1 (for a different proof, see [5]).

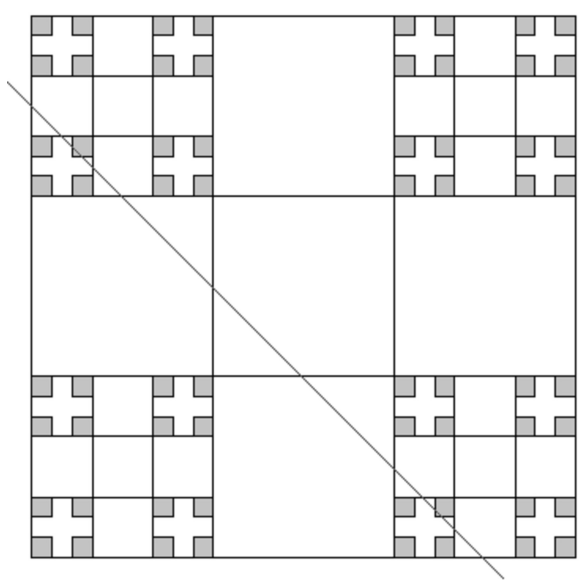

FIGURE 1.

(C) 2016 Mathematical Institute, Slovak Academy of Sciences. 2010 Mathematics Subject Classification: 28A78, 28 A 80.

Keywords: Cantor sets, Ana sets, thickness, sums of sets. 
In this paper, we concentrate on another small set, the Golden Ana set. We start with the Ana set which is based on a verbal sequence. We begin with the letter "a" in stage 0 . In stage 1 , we replace the "a" with "ana". In the subsequent stages every "a" is replaced by "ana" and every " $\mathrm{n}$ " is replaced by "ann". This is really just pronouncing each letter as, "An a", or "An n". The first four stages are:

$$
\begin{gathered}
\text { a } \\
\text { ana } \\
\text { ana ann ana } \\
\text { ana ana ana ann ann ana ann ana }
\end{gathered}
$$

In the article by $\mathrm{Pe}$ [4 he answers the question, "At stage $k$, how many a's and n's are there?" $\left(\left(3^{k-1}+1\right) / 2\right.$ a's and $\left(3^{k-1}-1\right) / 2$ n's, making $3^{k-1}$ total letters). He also introduces a new sequence by bringing up the following misconception: Since $\mathrm{n}$ is a consonant, then perhaps it should be said as, "A n". Thus the new sequence starts with "a" and there after every "a" is replaced with "ana" while every " $n$ " is replaced by "a n". The first four stages here are then

$$
\begin{gathered}
\text { a } \\
\text { ana } \\
\text { ana an ana } \\
\text { ana an ana ana an ana an ana }
\end{gathered}
$$

$\mathrm{P}$ e then shows that at stage $k$ the number of a's in a Golden Ana set is $a(k)=F(2 k-1)$ and the number of n's is $n(k)=F(2 k-2)$ where $F(k)$ is the $k$ th Fibonacci number. We then have

$$
\frac{a(k)}{n(k)}=\frac{F(2 k-1)}{F(2 k-2)} \rightarrow \phi=\frac{1+\sqrt{5}}{2},
$$

the Golden Ratio, hence calling the sequence the Golden Ana sequence.

We now turn this set into a set in $[0,1]$. We do this in the natural manner as is done with the Cantor set. For the Ana set, stage $k$, subdivide the unit interval into $3^{k-1}$ subintervals there at that stage there are $3^{k-1}$ letters. Moving left to right, if the $j$ th letter in stage $k$ is an a, do nothing. If it is an n, remove the $j$ th open subinterval. This gives us

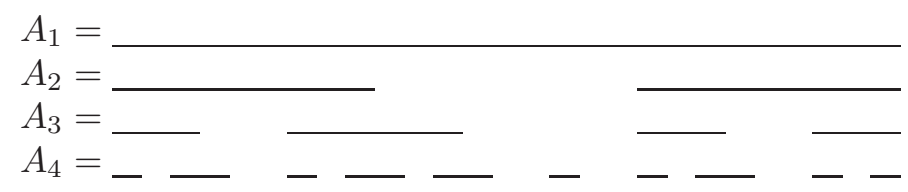

Doing the same with the Golden Ana set the first four iterates are

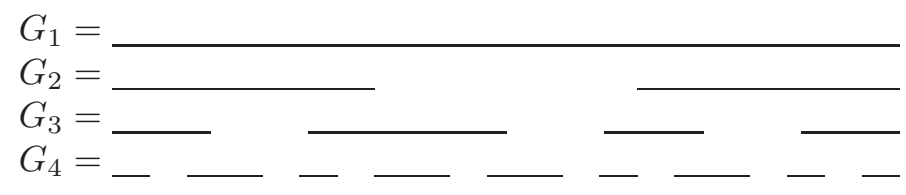


Corresponding to these collections of intervals there are two sets we are interested in observing. These are

and

$$
A=\bigcap_{k=1}^{\infty} A_{k}, \quad \text { called the Ana set }
$$

$$
G=\bigcap_{k=1}^{\infty} G_{k}, \quad \text { called the Golden Ana set. }
$$

For this paper, we will concentrate on $G$ and remark a little on $A$. However we do note here, there are four other interesting sets we can develop:

$$
\begin{aligned}
& \bar{A}=\limsup _{k \rightarrow \infty} A_{k}=\bigcap_{k=1}^{\infty}\left(\bigcup_{m=k}^{\infty} A_{m}\right), \\
& \underline{A}=\liminf _{k \rightarrow \infty} A_{k}=\bigcup_{k=1}^{\infty}\left(\bigcap_{m=k}^{\infty} A_{m}\right), \\
& \bar{G}=\limsup _{k \rightarrow \infty} G_{k}=\bigcap_{k=1}^{\infty}\left(\bigcup_{m=k}^{\infty} G_{m}\right),
\end{aligned}
$$

and

$$
\underline{G}=\liminf _{k \rightarrow \infty} G_{k}=\bigcup_{k=1}^{\infty}\left(\bigcap_{m=k}^{\infty} G_{m}\right) .
$$

We change course now to look at two different points of view for the construction of the general idea of Cantor sets. Our first way will be what we refer to as the standard construction of a Cantor set.

Let $\left\{a_{n}\right\}$ be a sequence of values where $0<a_{n}<1 / 2$. Stage zero of the construction consists of the closed interval $I$. At stage $n$ we have $2^{n}$ closed intervals $I_{n, k}$ of length

$$
\left(a_{1} \times a_{2} \times \cdots \times a_{n}\right)|I| .
$$

In the next step each sub-interval $I_{n \times k}$ is divided into three pieces

$$
I_{n, k}^{0}, O_{n, k}, \quad \text { and } I_{n, k}^{1},
$$

where $O_{n, k}$ is an open interval between $I_{n, k}^{0}$ and $I_{n, k}^{1}$ and $I_{n, k}^{0}\left(I_{n, k}^{1}\right)$ has the same left (right) endpoints as $I_{n, k}$ and

$$
\frac{\left|I_{n, k}^{0}\right|}{\left|I_{n, k}\right|}=\frac{\left|I_{n, k}^{1}\right|}{\left|I_{n, k}\right|}=a_{n}
$$

The Cantor set is then

$$
C=C_{a_{n}}=\bigcap_{n=1}^{\infty}\left(\bigcup_{k=1}^{2^{n}} I_{n, k}\right)
$$


The second construction is as follows and comes from [3]. A Cantor set is any set of the form

$$
I \backslash\left(\bigcup_{i=1}^{\infty} O_{i}\right),
$$

where $I$ is a bounded, closed interval and $\left\{O_{i}\right\}$ is a countable collection of disjoint open intervals which have no endpoint in common and whose union is dense in $I$.

Our non-standard construction inductively defines $C$ in the following manner. At the zeroth level we have the interval $I$. Suppose our tree has been defined to the $n$th level. Let $I^{w}$ be an interval from that level. Let $O_{I} w$ be the interval in $\left\{O_{i}: i \geq 1\right\}$ of least index which is contained in $I^{w}$. Then we have

$$
I^{w}=I^{w 0} \cup O_{I^{w}} \cup I^{w 1} .
$$

Continue this process indefinitely to arrive at

$$
C=\bigcap_{n=0}^{\infty}\left(\bigcup_{|w|=n} I^{w}\right)
$$

This is referred to as a derivation of the Cantor set $C$ from $I$. The intervals $I, I^{0}, \ldots$ are called the bridges of the derivation, while the open intervals $O_{I}, O_{I^{0}}, \ldots$ are called the gaps of $C$.

Thickness is a method of looking at the size of the Cantor set. First seen in [3] it looks at the ratio between the sizes of the remaining subintervals and the gap removed to create those subintervals.

Definition 1. Let $C$ be a Cantor set. We define the thickness of $C, \tau(C)$, to be

$$
\tau(C)=\sup _{\mathcal{D}}\left\{\inf _{A \in \mathcal{D}}\left\{\min \left\{\frac{\left|A^{0}\right|}{\left|O_{A}\right|}, \frac{\left|A^{1}\right|}{\left|O_{A}\right|}\right\}\right\}\right\},
$$

where the supremum is over all derivations $\mathcal{D}$ of $C$ and the infimum is over all bridges $A$ of $D$.

Despite the myriad of derivations, there is a way to determine the thickness of a set using the idea of an ordered derivation.

Definition 2. A derivation is considered ordered if for intervals $A$ and $B$ in the derivation with $A=A^{0} \cup O_{A} \cup A^{1}, B=B^{0} \cup O_{B} \cup B^{1}$, and $A \subset B$, then

$$
\left|O_{A}\right| \leq\left|O_{B}\right| \text {. }
$$

This gives us the following theorem from Astels [1].

Theorem 1. If $\mathcal{D}_{O}$ is an ordered derivation, then

$$
\tau_{\mathcal{D}}(C) \leq \tau_{D_{O}}(C)
$$

where $\mathcal{D}$ is any derivation. 
As an application of Theorem 1 we can easily see the ordinary Cantor Set, $C$, has thickness 1 .

Astels' paper has the following application concerning thickness and the sums of Cantor sets. This states that if two Cantor sets are sufficiently thick, then their sum is the sum of the intervals from which they were derived.

Theorem 2. For $j=1$ or $j=2$, let $C_{j}$ be a Cantor set derived from $I_{j}$ with $O_{j}$ a gap of maximal size in $C_{j}$. Assume that

$$
\left|O_{1}\right| \leq\left|I_{2}\right| \text { and }\left|O_{2}\right| \leq\left|I_{1}\right| \text {. }
$$

If $\tau\left(C_{1}\right) \times \tau\left(C_{2}\right) \geq 1$, then $C_{1}+C_{2}=I_{1}+I_{2}$.

This is another way of proving that the sum of the Cantor Set with itself is the interval $[0,2]$.

\section{Results}

The Ana Set is the ordinary Cantor set. Our interest is to look at the sum of Golden Ana sets for something similar to $A+A=[0,2]$. We would like the sum of copies of $G$ to be an interval. That is not the case with just two copies of the set.

Theorem 3. The sum $G+G \neq[0,2]$. The sum is not an interval.

P r o o f. At the second stage of the construction we have four intervals: $[0,1 / 8]$, $[1 / 4,1 / 3],[2 / 3,3 / 4]$, and $[7 / 8,1]$. Thus $G+G$ misses all of the points between $1 / 8+1 / 3$ and $1 / 4+1 / 4$; that is, the interval $(11 / 24,1 / 2)$.

In thickness terms, this tells us that the Golden Ana set should have thickness strictly less than 1 . There is a problem in measuring the thickness of the Golden Ana set. If we look at the iterates $G_{2}$ and $G_{4}$ we see that their intersection leaves the isolated point $1 / 3$. The work by J o n es [2] shows that the end result $G$ will have isolated points. Thus $\tau$ is not defined for $G$ (again, if we allow isolated points, we would have $\tau(G)=0$ which is equally problematic).

The issue of allowing isolated points is not important for our real goal which is to look at the sum of Golden Ana Sets. So we create the set $G^{*}$, the set of all non-isolated points in the Golden Ana set; that is,

$$
G^{*}=\{x \mid x \in G, x \text { is not isolated }\} .
$$

Since $G$ is uncountable and any set of isolated points is countable, we know that $G^{*}$ is nonempty. With the isolated points removed this does fit the definition of a Cantor set from Newhouse. With regards to $G^{*}$ we have the theorem below. 
TheOREM 4. The set $G^{*}$ has positive thickness.

Proof. At stage $k-1$ in the construction of $G$, the unit interval has been divided into intervals of length $\frac{1}{F_{2(k-1)}}$. So at that stage we look at a closed subinterval, call it $A$. At the next step, an open subinterval $O_{A}$ of length $\frac{1}{F_{2 k}}$ is removed from $A$. With regards to the creation of $G^{*}$ the interval $O_{A}$ may have isolated points as endpoints and so the interval removed to create $G^{*}$ may be even larger. Since $G^{*}$ is nonempty there is a limit to how much extra length will be removed. The amount added will be at most $\frac{1}{3} \frac{1}{F_{2 k}}$ on both sides of $O_{A}$ because

$$
\frac{F_{2 k}}{F_{2 k-2}} \leq 3
$$

This gives the ratio in the determination of the thickness as bounded below by

$$
\frac{\frac{1}{F_{2 k}}-\frac{1}{3} \frac{1}{F_{2 k}}}{\frac{1}{F_{2 k}}+\frac{2}{3} \frac{1}{F_{2 k}}}=\frac{2 / 3}{5 / 3}=\frac{2}{5} .
$$

And so we have our desired result.

However, we can get a positive answer if we include more copies of the set. To show this we must now introduce the idea of normalized thickness [1] and a theorem concerning this type of thickness.

Definition 3. For any Cantor set, the normalized thickness of $C$ is given by

$$
\gamma(C)=\frac{\tau(C)}{1+\tau(C)} .
$$

Applying this to $G^{*}$ we have $\gamma\left(G^{*}\right)>2 / 7$.

Theorem 5 (from [1]). Let $k$ be a positive integer and for $j=1,2, \ldots, k$, let $C_{j}$ be a Cantor set derived from $I_{j}$ with $O_{j}$ a gap of maximal size in $C_{j}$. Let $S_{\gamma}=\gamma\left(C_{1}\right)+\gamma\left(C_{2}\right)+\cdots+\gamma\left(C_{k}\right)$. If

$$
\begin{aligned}
\left|I_{r+1}\right| & \geq\left|O_{j}\right| \quad \text { for } r=1, \ldots, k-1, \quad \text { and } j=1, \ldots, r, \\
\left|I_{1}\right|+\left|I_{2}\right|+\cdots+\left|I_{r}\right| & \geq\left|O_{r+1}\right| \text { for } r=1, \ldots, k-1,
\end{aligned}
$$

and

$$
S_{\gamma} \geq 1
$$

then

$$
C_{1}+C_{2}+\cdots+C_{k}=I_{1}+I_{2}+\cdots+I_{k}
$$

So our positive result is

TheOREM 6. The sum of four Golden Ana sets is an interval. In fact,

$$
G+G+G+G=[0,4] .
$$




\section{SUMS OF GOLDEN ANA SETS}

P r o o f. This is an application of the result above to $G^{*}$ along with the fact that if $A \subset B$, then

$$
A+A \subset B+B \text {. }
$$

Open question. Where does $G+G+G$ fit in on this? Is this the interval $[0,3]$ or are there points in the interval, but not in the sum?

\section{REFERENCES}

[1] ASTELS, S.: Thickness measures for Cantor sets, Electron. Res. Announc. Amer. Math. Soc. 5 (1999), 108-111.

[2] JONES, C.: (private communication)

[3] NEWHOUSE, S.: The abundance of wild hyperbolic sets and nonsmooth stable sets for diffeomorphisms, Publ. Math. Inst. Hautes Études Sci. 50 (1979), 101-151.

[4] PE, J.: Ana's golden fractal, Fractals 11 (2003), 309-313.

[5] VALLIN, R. W.: The Elements of Cantor Sets-With Applications. With a foreword by David M. Bressoud. J. Wiley \& Sons, Inc., Hoboken, NJ, 2013.

E-mail: robert.vallin@lamar.edu 